\title{
Caracterización preliminar de la inmunidad pasiva natural en granjas porcicolas y evaluación de un sistema para incrementar la transferencia de anticuerpos
}

\author{
Ángela Benavides ${ }^{1}$, Jorge Almansa $\mathrm{MSc}^{2}$, Alfonso Caldrón $\mathrm{MSc}^{3}$, Orlando Torres MSc., Nohora Delgado MSc ${ }^{3}$ y Gustavo García PhD ${ }^{3}$ \\ 'Microbiología Agrícola y Veterinaria, Facultad de Ciencias, Pontificia Universidad Javeriana, ${ }^{2}$ Universidad Antonio Nariño \\ y ${ }^{3}$ Programa Nacional de salud Animal, Corpoica \\ Correspondencia: jorge.almansa@uan.edu.co \\ Recibido: 15-05-05 / Aceptado: 15-11-05
}

\section{Resumen}

Se determinó la transferencia pasiva de inmunidad natural en lechones de dos granjas porcícolas comerciales. Para ello se tomaron muestras de sangre a 221 lechones a las 24 horas de nacidos y a 21 cerdas madres. Los lechones de cada granja fueron divididos en dos grupos uno experimental y uno control. El primer grupo se manejo de acuerdo con las normas usuales para cada granja, mientras que a las madres de los lechones del segundo grupo se les aplicó un producto inmunomodulador a base de LPS y Propionibacterium granulosum. Los sueros obtenidos fueron analizados por las técnicas de electroforesis sobre acetato de celulosa, inmunoelectroforesis, inmunodifusión radial cuantitativa y prueba de turbidez con sulfato de Zinc. La concentración de proteína total fue determinada con el refractómetro de Golberg. Se analizan los resultados obtenidos y se discute la importancia de la adecuada transferencia pasiva de inmunidad natural y el posible uso del inmunomodulador en granjas comerciales con problemas de morbimortalidad neonatal.

Palabras clave: Inmunidad pasiva en porcinos, anticuerpos pasivos, concentración de inmunoglobulinas, calostro, inmunomoduladores.

\footnotetext{
Abstract

Passive natural immunity transfer in pigs from two different commercial breeding farms was determined. For this purpose, blood from 221 pigs, 24 hours after being born and 21 mothers pigs, were taken. The pigs from each farm were separated into two groups consisting of approximately 50 individuals each. The first group was treated as usually done by the farm owners, and to the second, an immunomodulator product (produced with LPS), was to the mothers given. Obtained serums were by means of cellulose acetate electrophoresis, immunoelectrophoresis, quantitative radial immunodiffusion and zinc sulfate turbidity tests analyzed. Total protein concentration was determined using a Golberg refractometer. Obtained results were analyzed and the importance of an adequate natural passive immunity transfer is discussed. Also discussed is the possible use of the immunodulator in commercial pig breeding farms that present high rates of neonatal morbimortality.

Key words: Passive immunity in pigs, passives antibodies, immunoglobulins concentrations, colostrum, immunomodulators.
} 


\section{Introducción}

Al nacer, los porcinos son en general, agammaglobulinémicos $(1,2)$, por lo que la transferencia pasiva de inmunidad desde la madre a través del calostro, reviste gran importancia $(1,3,4)$. Desde hace tiempo se conoce la relación entre la concentración de anticuerpos pasivos en el suero sanguíneo de los lechones a las 24 horas del nacimiento y su estado de salud hasta el destete $(3,5,6)$. De otro lado, factores tales como debilidad, hipoxia, orden de nacimiento y peso corporal pueden influenciar la toma de calostro y el posterior desarrollo de los animales $(2,7,8)$. En condiciones naturales el calostro materno es la única fuente de inmunoglobulinas disponible para el cerdo recién nacido. Estas proteínas globulares, predominantemente Ig G, son absorbidas en intestino y llevadas al torrente circulatorio dentro de las primeras 24 horas luego del nacimiento $(2,9)$. De esta manera el consumo de calostro inmediatamente después del parto, resulta esencial por dos razones; primero, la concentración de inmunoglobulinas calostrales declinan rápidamente después del parto, y dos, la capacidad del intestino del neonato para absorber tales inmuno-globulinas se pierde pocas horas después del nacimiento. Se ha determinado previamente que las concentraciones menores de $10 \mathrm{mg} / \mathrm{ml}$ de $\mathrm{IgG}$ sanguínea de lechones a las 24 horas del nacimiento, corresponden a fallas de diversa índole en la transferencia pasiva de inmunidad y que tales animales presentan una probabilidad elevada de enfermar y/o morir antes del destete $(9,10)$.

En el presente trabajo se evaluó la inmunidad pasiva natural en dos piaras comerciales y se estableció su relación con el estado de salud de los animales hasta el momento del destete. También se estudió el impacto de la administración de un inmunomodulador $(11,12)$ a las madres, durante el último tercio de la gestación, con el fin de aumentar los niveles de inmunoglobulinas en calostro e incrementar así su absorción por parte de los lechones.

\section{Materiales y métodos}

\section{Población de Estudio.}

La población del estudio pertenecía a dos granjas porcícolas comerciales, la primera ubicada en el municipio de Mosquera (Cundinamarca) (Granja 1) y la segunda en el municipio de La Mesa (Cundinamarca) (Granja 2). En cada granja se conformaron dos grupos: a) lechones sin tratamiento (control) y b) lechones de madres tratadas con un inmunomodulador a base de lipopolisacárido (LPS) de E. coli y células inactivadas de Propionibacterium granulosum. El producto se aplicó 8 y 2 días antes del parto en un volumen de 5 y $2 \mathrm{ml}$ por animal por vía intramuscular. Se incluyeron cerdas de 1 hasta 8 partos cuyas camadas estuvieron entre 8 y 12 lechones.

\section{Muestras}

Las muestras estudiadas fueron sueros provenientes de 221 lechones con aproximadamente 24 horas de nacidos y sueros de cerdas madres (21). La conservación de estos sueros se realizó por congelación a $-40^{\circ} \mathrm{C}$ hasta el momento de la realización de las pruebas. Todas las muestras fueron corridas por las técnicas de electroforesis sobre acetato de celulosa $(7,10,13,14$, 15), refractometría (10), prueba de turbidez con sulfato de zinc (10), inmunodifusión radial cuantitativa para IgG e inmunoelectroforesis $(10,14,15,16)$.

\section{Electroforesis en Acetato de Celulosa.}

Las muestras obtenidas fueron corridas por electroforesis en tiras de acetato de celulosa siguiendo el procedimiento indicado para el sistema Sepratek de Gelman Sciences, inc. y posteriormente aclaradas siguiendo el protocolo propuesto por Morilla \& Bautista (10). Las placas fueron leídas en un densitómetro Shimadzu CS-9000. 
Tabla 1. Distribución muestral de lechones en las granjas y grupos estudiados.

\begin{tabular}{ccccc}
\hline \multirow{2}{*}{ GRUPO } & & FRECUENCIA & $\%$ \\
& & & & \\
\hline \multirow{2}{*}{ EXPTAL } & \multirow{2}{*}{ Valid } & GRNJA 1 & 55 & 50,9 \\
& & GRNJA 2 & 53 & 49,1 \\
\cline { 3 - 5 } & & TOTAL & 108 & 100 \\
\hline \multirow{2}{*}{ CONTROL } & \multirow{2}{*}{ Valid } & GRNJA 1 & 53 & 46,9 \\
& & GRNJA 2 & 60 & 53,1 \\
\cline { 3 - 5 } & & TOTAL & 113 & 100 \\
\hline
\end{tabular}

\section{Refractomería.}

La concentración de proteína total en los sueros fue estimada con el refractómetro de Golberg (marca CETI ref: 8100-0120 DIGIT- 012).

\section{Prueba de Turbidez con Sulfato de Zinc.}

Esta técnica se llevó a cabo utilizando el protocolo propuesto por Morilla y Bautista (10). Las muestras se analizaron empleando un espectrofotómetro (MILTON ROY Spectronic 601). Las muestras precipitadas fueron leídas a $660 \mathrm{~nm}$ y los resultados se expresaron en unidades de turbidez del sulfato de zinc (unidades TSZ).

\section{Inmunodifusión Radial Cuantitativa.}

Las inmunodifusiones se realizaron siguiendo protocolos Morilla y Bautista (10), Weir D. Las apropiadas diluciones de anti-inmunoglobulina G Porcinas se evaluaron en pruebas previas con sueros que mostraron diferente concentración en las pruebas de turbidez con sulfato de zinc (16).

\section{Inmunoelectroforesis.}

Para realizar esta prueba se seleccionaron sueros de acuerdo con los resultados obtenidos para proteína total, se corrieron muestras con concentraciones altas, bajas e intermedias y se contrastaron con los sueros maternos, adicionalmente se evaluó el antisuero total porcino producido. Se utilizaron los protocolos propuestos por Rodríguez et al. (17) y Morilla y Bautista (14).

\section{Antisueros policlonales contra suero total porcino e IgG porcina.}

La producción del antisuero se realizó en conejos, empleando protocolos de inmunización propuestos por Rodríguez, et al. (17) y Morilla y Bautista (14). Como inóculo se utilizaron sueros de porcinos adultos sanos e IgG porcina pura (Sigma I4381).

\section{Resultados}

La tabla 1 contiene la distribución de las muestras, tanto por granja como por grupos control y experimental en cada una de ellas. Esta tabla, como todas las referentes al análisis estadístico presentadas en el presente trabajo, se muestra de acuerdo con el formato contenido en el Programa Estadístico para Ciencias Sociales (SPSS), con el cual se analizaron los resultados.

Las condiciones de comparación de pesos al nacimiento y al destete entre los grupos experimental y control, en cada una de las dos granjas estudiadas se presentan en la tabla 2. Nótese que el grupo experimental presentó un mayor peso en ambas circunstancias.

En la tabla 3 se muestra la comparación de pesos obtenidos por los lechones al nacimiento y al destete, en donde se aprecia la diferencia significativa entre

Tabla 2. Comparación de pesos de lechones al nacimiento y al destete entre las dos granjas estudiadas.

\begin{tabular}{llrrr}
\hline GRUPO & & \multicolumn{2}{c}{ PESO NCTO } & PESO DSTTE \\
\hline & N & Presentes & 108 & 107 \\
& Media & Ausentes & 0 & 1 \\
& Error estand.media & 1668,52 & 6766,36 \\
& Desviación estándar & 26,33 & 144,98 \\
& rango & 273,66 & 1499,68 \\
& Mínimo & 1300 & 5900 \\
EXPTAL & Máximo & 1000 & 4100 \\
\hline & & Presentes & 113 & 10000 \\
& N & Ausentes & 0 & 95 \\
& Media & 1551,33 & 6388,42 \\
& Error estand.media & 28,08 & 175,22 \\
& Desviación estándar & 298,54 & 1707,8 \\
& Rango & 1600 & 8300 \\
& Mínimo & 800 & 3200 \\
CONTROL & Máximo & 2400 & 11500 \\
\hline
\end{tabular}




\begin{tabular}{|c|c|c|c|c|c|c|}
\hline & & $\begin{array}{c}\text { Suma } \\
\text { Cuadrados }\end{array}$ & df & $\begin{array}{l}\text { Media } \\
\text { Cuadr. }\end{array}$ & $F$ & Sig. \\
\hline \multirow[t]{3}{*}{ PESO NCTO } & Entre Grupos & 758401,310 & 1,000 & 758401,310 & 9,230 & 0,003 \\
\hline & Dentro Grupos & 17995263,848 & 219,000 & 82170,155 & & \\
\hline & Total & 18753665,158 & 220,000 & & & \\
\hline \multirow[t]{3}{*}{ PESO DSTTE } & Entre Grupos & 7187670,219 & 1,000 & 7187670,219 & 2,805 & 0,096 \\
\hline & Dentro Grupos & 512556141,663 & 200,000 & 2562780,708 & & \\
\hline & Total & 519743811,881 & 201,000 & & & \\
\hline
\end{tabular}

pesos al nacimiento $(\mathrm{P}<0.005)$ pero no al destete, entre los grupos con tratamiento y control respectivamente, en las granjas 1 y 2 . En la figura 1, pesos al nacimiento, se observa que la longitud de las barras verticales, que representan las desviaciones estándar de la variable en los dos grupos, no se sobreponen, lo cual corrobora la diferencia significativa presentada entre los grupos experimental y control, a favor del primero (promedios de pesos al nacimiento en grupo experimental 1.668 gr y en grupo control 1.551 gr.). A pesar de que en el estudio se estableció una diferencia de peso al destete (Figura 2) a favor del grupo experimental, la diferencia no es estadísticamente significativa (promedios de pesos al destete en grupo experimental 6.766 gr y en grupo control 6.388 gr.).

La tabla 4 enumera las condiciones del análisis de varianza de los datos obtenidos para concentración de proteína total, albúmina, globulinas, IgG por inmunodifusión radial cuantitativa (IDRC) y test de turbidez con sulfato de zinc.

De otra parte la tabla 5 muestra los resultados obtenidos en los análisis de varianza con los datos de la tabla anterior. En ella se aprecia que existen diferencias significativas a favor de los grupos tratados, en concentración total de proteína sérica, albúmina y en los resultados obtenidos con el test de turbidez con sulfato de zinc, mientras que para la concentración de globulinas, así como para los resultados obtenidos por IDRC, las diferencias, a pesar de presentarse a favor de los grupos tratados, no son significativas.

La tabla 6 indica la prueba de $\mathrm{T}$ para las distintas variables analizadas. Las figuras 3,4,5,6 y 7 representan las diferencias para proteína total, albúmina, globulinas, IDRC, y turbidez con sulfato de zinc respectivamente.

Dado que algunas de las muestras tomadas presentaron diferentes grados de hemólisis, evaluadas subjetivamente como una cruz $(+)$, dos cruces $(++)$, tres cruces $(+++)$ y cuatro cruces $(++++)$, de acuerdo con el grado de color mostrado por el suero, fue necesario establecer si la misma incidía en los resultados obtenidos. La figura 8 muestra un histograma de la forma como se distribuyó la hemólisis y sus distintos grados en las muestras de los dos grupos. Aquí se puede ver que la hemólisis se distribuyó de manera homogénea entre todos los 4 grupos del trabajo, lo que explicaría el hecho de que no incida en los resultados obtenidos.

La figura 9 corresponde a un histograma donde se representan los porcentajes de individuos del estudio que no enfermaron, murieron, enfermaron y enfermaron y murieron.

En la figura 10 se grafican los porcentajes de individuos que dentro de cada grupo presentaron diarrea, murieron por aplastamiento, murieron a la toma de sangre, presentaron baja vitalidad, inanición, asfixia u otra. Las figuras 11 y 12 muestran histogramas que grafican la mortalidad observada en los grupos experimentales y controles de las granjas 1 y 2 respectivamente.

\section{Obtención y prueba de los antisueros.}

Los sueros hiperinmunes obtenidos a partir de los animales inmunizados fueron probados por Inmunoelectroforesis. Ambos esquemas condujeron a antisueros de buena calidad, ya que permiten visualizar 
Tabla 4. Análisis de las diferentes variables en lechones entre grupos experimentales y controles de las dos granjas.

\begin{tabular}{|c|c|c|c|c|c|c|c|c|c|}
\hline & & \multirow[t]{2}{*}{$\mathbf{N}$} & \multirow[t]{2}{*}{ Media } & \multirow[t]{2}{*}{$\begin{array}{l}\text { Desvia. } \\
\text { Estándar }\end{array}$} & \multirow[t]{2}{*}{ Error. Est. } & \multicolumn{2}{|c|}{$\begin{array}{l}95 \% \text { Intervalo de } \\
\text { Confianza - Media }\end{array}$} & \multirow[t]{2}{*}{ Mínimo } & \multirow[t]{2}{*}{ Máximo } \\
\hline & & & & & & $\begin{array}{l}\text { Límite más } \\
\text { bajo }\end{array}$ & $\begin{array}{l}\text { Límite más } \\
\text { más alto }\end{array}$ & & \\
\hline & EXPTAL & 108,000 & 6,2640 & 0,8690 & $8,365 \mathrm{E}-02$ & 6,098 & 6,43 & 1,800 & 8,500 \\
\hline \multirow[t]{3}{*}{ PROT. TTAL } & CONTROL & 113,000 & 5,9540 & 0,8310 & $7,8140 \mathrm{E}-02$ & 5,799 & 6,109 & 3,000 & 8,500 \\
\hline & TOTAL & 221,000 & 6,1050 & 0,8620 & $5,7980 \mathrm{E}-02$ & 5,991 & 6,22 & 1,800 & 8,500 \\
\hline & EXPTAL & 108,000 & 0,94097 & 0,3177 & $3,0566 \mathrm{E}-02$ & 0,88038 & 1,00156 & 0,326 & 2,588 \\
\hline \multirow[t]{3}{*}{ ALBUMINA } & CONTROL & 113,000 & 0,82921 & 0,2591 & $2,4374 \mathrm{E}-02$ & 0,78092 & 0,87751 & 0,260 & 2,093 \\
\hline & TOTAL & 221,000 & 0,88383 & 0,2939 & 1,9771E-02 & 0,84486 & 0,92279 & 0,260 & 2,588 \\
\hline & EXPTAL & 108,000 & 5,32944 & 0,8237 & $7,9263 \mathrm{E}-02$ & 5,17232 & 5,48657 & 1,342 & 7,331 \\
\hline \multirow[t]{3}{*}{ GLOBULINAS } & CONTROL & 113,000 & 5,11967 & 0,8491 & 7,9873E-02 & 4,96141 & 5,27793 & 2,590 & 7,713 \\
\hline & TOTAL & 221,000 & 5,22219 & 0,8415 & $5,6603 \mathrm{E}-02$ & 5,11063 & 5,33374 & 1,342 & 7,713 \\
\hline & EXPTAL & 108,000 & 1,0409 & 0,2120 & $2,0400 \mathrm{E}-02$ & 1,0004 & 1,0813 & 0,000 & 1,600 \\
\hline \multirow[t]{3}{*}{ R. IDRC } & CONTROL & 113,000 & 1,0107 & 0,2247 & $2,1140 \mathrm{E}-02$ & 0,9688 & 1,0526 & 0,000 & 1,300 \\
\hline & TOTAL & 221,000 & 1,0255 & 0,2186 & $1,4700 \mathrm{E}-02$ & 0,9985 & 1,0544 & 0,000 & 1,600 \\
\hline & EXPTAL & 108,000 & 7,1345 & 1,9501 & 0,1876 & 6,7626 & 7,05065 & 0,010 & 15,680 \\
\hline \multirow[t]{2}{*}{ SULF. ZINC } & CONTROL & 112,000 & 5,9423 & 1,7063 & 0,1612 & 5,6228 & 6,2618 & 0,030 & 11,930 \\
\hline & TOTAL & 220,000 & 6,5276 & 1,9211 & 0,1295 & 6,2723 & 6,7029 & 0,010 & 15,680 \\
\hline
\end{tabular}

Tabla 5. Análisis de varianza de las variables en lechones de los grupos y granjas estudiadas.

\begin{tabular}{rlrrrrr}
\hline & \multicolumn{1}{c}{$\begin{array}{c}\text { Suma } \\
\text { Cuadrados }\end{array}$} & \multicolumn{1}{c}{ df } & \multicolumn{1}{c}{$\begin{array}{l}\text { Media } \\
\text { Cuadr. }\end{array}$} & F & Sig. \\
\hline PROT. TTAL & Entre Grupos & 5,304 & 1 & 5,304 & 7.344 & .007 \\
& Dentro Grupos & 158,150 & 219 & 0,722 & & \\
& Total & 163,453 & 220 & & & \\
ALBUMINA & Entre Grupos & 0,690 & 1 & 0,69 & 8.247 & .004 \\
& Dentro Grupos & 18,315 & 219 & $8,36 \mathrm{E}-02$ & & \\
& Total & 19,005 & 220 & & & \\
GLOBULINAS & Entre Grupos & 2,430 & 1 & 2,43 & 3.470 & .064 \\
& Dentro Grupos & 153,343 & 219 & 0,7 & & \\
& Total & 155,773 & 220 & & & \\
IDRC & Entre Grupos & $0,05009 \mathrm{E}-2$ & 1 & $5,01 \mathrm{E}-02$ & 1.048 & .307 \\
& Dentro Grupos & 10,463 & 219 & $4,78 \mathrm{E}-02$ & & \\
& Total & 10,513 & 220 & & & \\
SULF. ZINC & Entre Grupos & 78,150 & 1 & 78,15 & 23.336 & .000 \\
& Dentro Grupos & 730,064 & 218 & 3,349 & & \\
& Total & 808,214 & 219 & & & \\
\hline
\end{tabular}

de manera adecuada las bandas constituidas por los distintos grupos de proteínas séricas. Los antisue-ros contra suero porcino total fueron utilizados para contrastar algunos de los resultados obtenidos de lechones con diferentes concentraciones de inmunoglobulinas y el antisuero anti IgG porcina se probó por IEF a fin de determinar su actividad y pureza y luego se empleó en las pruebas de inmuno-difusión radial cuantitativa. El estudio comparativo de las madres de cada uno de los grupos de las dos granjas, no mostró diferencias estadísticamente significativas en cuanto a concentración de albúmina, globulinas, proteína total, IDRC ni test de turbidez con sulfato de zinc. Sin embargo las pruebas para concentración de globulinas, IDRC y turbidez con sulfato de zinc reflejan valores menores en el grupo experimental, lo que podría reflejar el paso de Igs maternas a los lechones a través del calostro.

www.unicolmayor.edu.co 


\section{Discusión}

El presente fue un estudio clínico, donde se analizaron animales de dos granjas, en las cuales se establecieron dos grupos, lechones cuyas madres recibieron tratamiento con un inmunomodulador preparado con LPS de E. coli y células inactivadas de Propionibacterium granulosum y un grupo control. (Tabla 1).

Los análisis de varianza de los pesos al nacer de lechones provenientes de madres tratadas y sin tra$\operatorname{tar}$ (Tabla 3) muestran una diferencia significativa a favor de los hijos de madres tratadas, lo cual podría estar indicando que el producto inmunomodulador posee propiedades estimuladoras indirectas sobre el crecimiento fetal. Como se sabe el LPS puede actuar uniendo inespecíficamente receptores de linfocitos B y T y el CD14 de macrófagos con lo cual estas células se activan iniciando, en el caso de los linfocitos, un proceso de blastogénesis $(2,9,19)$. Las células activadas secretan entonces una gama de citoquinas con diversas actividades a distintos niveles orgánicos que podrían pasar a los fetos a través de la placenta, estimulando su crecimiento durante la última semana de gestación. De otro lado, aun cuando resulta menos probable dada la constitución placentaria, el LPS podría alcanzar la circulación fetal y estimular directamente receptores involucrados con el crecimiento.

Al destete continua presentándose una diferencia de peso a favor de los lechones provenientes de hembras tratadas, pero la diferencia no resulta estadísticamente significativa.

Como se mencionó en el aparte de resultados se presentaron diferencias significativas en pro de los grupos experimentales en cuanto a proteína total evaluada por refractometría, concentración de albúminas determinada por la técnica de electroforesis sobre membranas de acetato de celulosa (EAC) y densitometría y los resultados obtenidos con el test de sulfato de zinc; mientras que para la variable concentración de globulinas determinada por la técnica de electroforesis sobre membranas de acetato de celulosa (EAC) y densitometría, así como para los
Tabla 6. Prueba de T para variables analizadas en lechones de los grupos y granjas estudiadas.

\begin{tabular}{lrlll}
\hline \multicolumn{1}{c}{ GRUPO } & N & Media & $\begin{array}{c}\text { Desviación } \\
\text { Est. }\end{array}$ & $\begin{array}{c}\text { Error Est. } \\
\text { Media }\end{array}$ \\
Exptal. & 108 & 1668.52 & 273.66 & 26.33 \\
Control & 113 & 1551.33 & 298.54 & 28.08 \\
Exptal. & 107 & 6766.36 & 1499.68 & 144.98 \\
Control & 95 & 6388.42 & 1707.80 & 175.22 \\
Exptal. & 108 & .94097 & .31765 & $3.0566 \mathrm{E}-2$ \\
Control & 113 & .82921 & .25910 & $2.4374 \mathrm{E}-2$ \\
Exptal. & 108 & 5.32944 & .82372 & $7.9263 \mathrm{E}-2$ \\
Control & 113 & 5.11967 & .84906 & $7.9873 \mathrm{E}-2$ \\
Exptal. & 108 & 1.0409 & .2120 & $2.040 \mathrm{E}-2$ \\
Control & 113 & 1.0107 & .2247 & $2.114 \mathrm{E}-2$ \\
Exptal. & 108 & 7.1345 & 1.9501 & .1876 \\
Control & 112 & 5.9423 & 1.7063 & .1612 \\
\hline
\end{tabular}

resultados obtenidos por IDRC, las diferencias aún cuando se presentan, no fueron significativas. Es importante anotar que debido a la interferencia sobre el electroforetograma provocada por la hemoglobina y otras proteínas de los glóbulos rojos lisados, no pudieron realizarse lecturas individuales de las fracciones alfa-beta-gamma de las globulinas, por lo que fue preciso recurrir a analizar la fracción globulínica de manera global, con lo cual la sensibilidad de la electroforesis se perdió para el estudio. Sin embargo, la prueba de turbidez con sulfato de zinc, permitió establecer de manera más clara las diferencias en cuanto a concentración de gammaglobulinas en los lechones hijos de madres tratadas, lo cual se había reportado ya por Álvarez, B (4) y por Ortiz, J.M. (12). Es importante anotar que la prueba de turbidez evalúa las concentraciones de IgG1, G2 y $\mathrm{M}$, mientras que la IDRC únicamente midió IgG1, tal como se observa en IEF donde se prueba el antisuero. La IDRC no permitió marcar un contraste mas claro entre las concentraciones específicas de IgG y aún cuando es apreciable una diferencia entre los grupos tratados y control a favor de los primeros, esta no es significativa. En el análisis general de las pruebas puede apreciarse que cuando las diferencias de concentración son grandes, estas se observan a simple vista. Sin embargo cuando las concentraciones son 


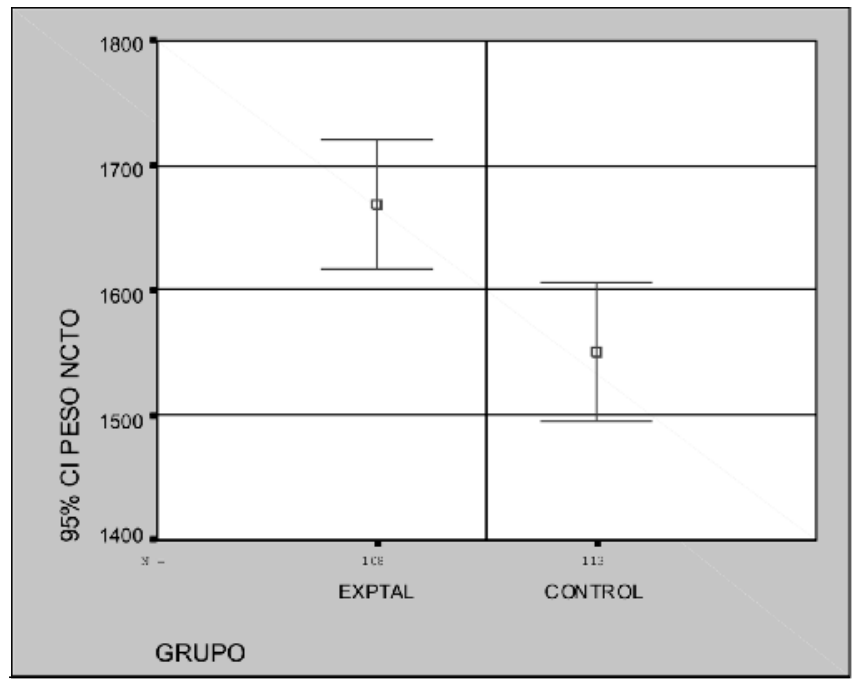

Figura 1. Diagrama comparativo de pesos de lechones al nacimiento entre grupos experimentales y controles de las dos granjas.

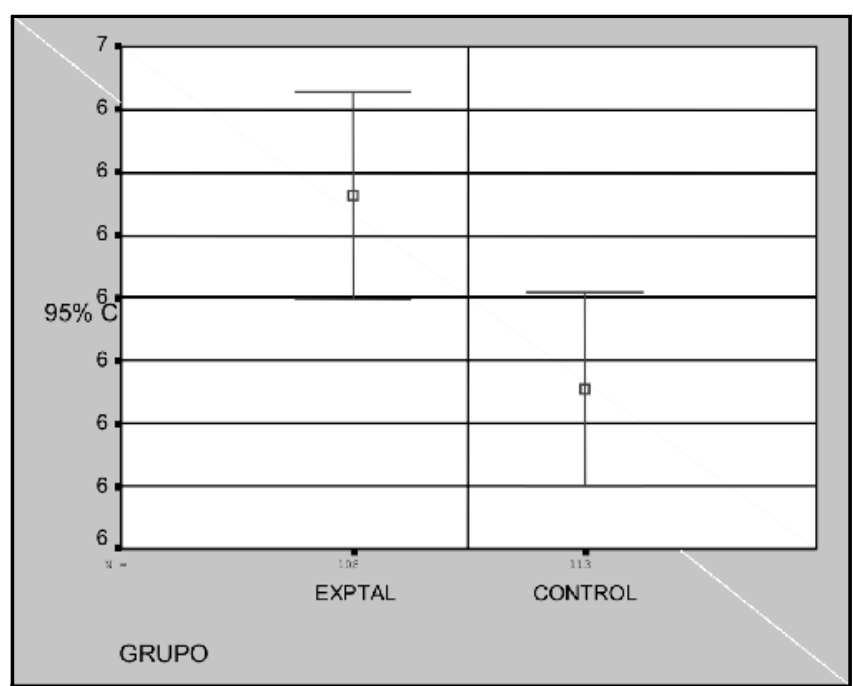

Figura 3. Diagrama comparativo en lechones de valores de proteína total entre grupos experimentales y controles de las dos granjas.

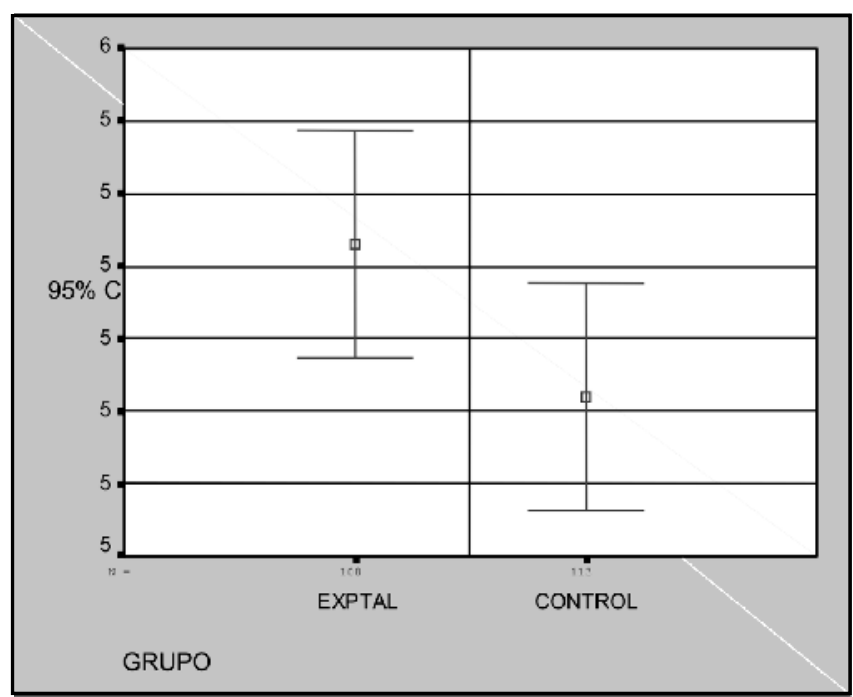

Figura 5. Diagrama comparativo en lechones de valores de globulinas entre grupos experimentales y controles de las dos granjas.

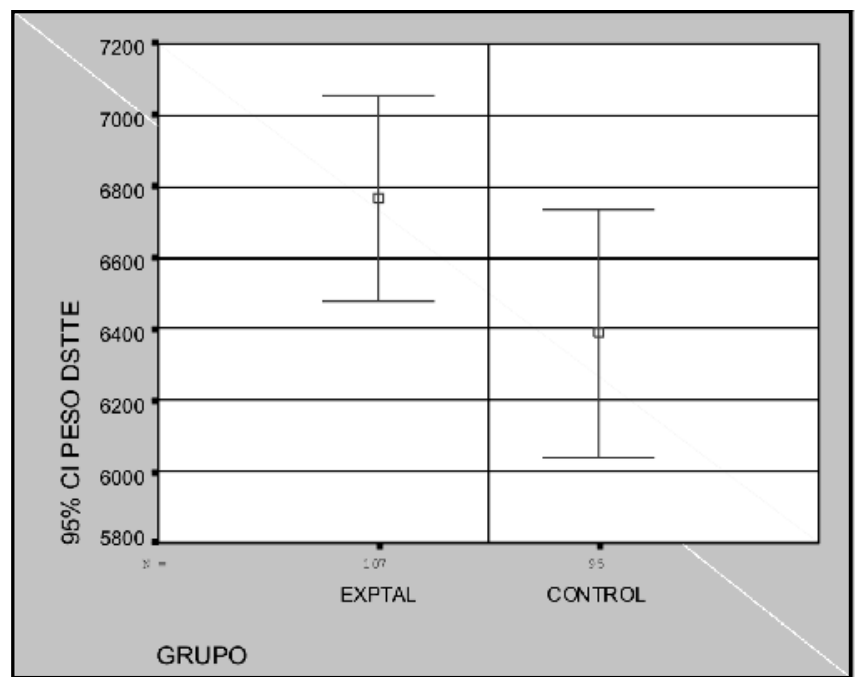

Figura 2. Diagrama comparativo de pesos de lechones al destete entre grupos experimentales y controles de las dos granjas.

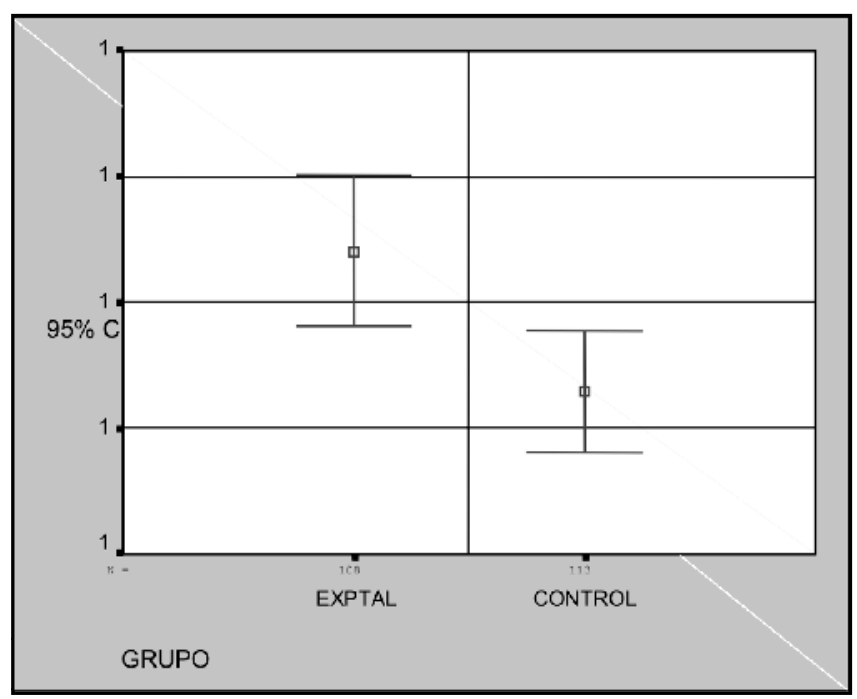

Figura 4. Diagrama comparativo en lechones de valores de albúmina entre grupos experimentales y controles de las dos granjas.

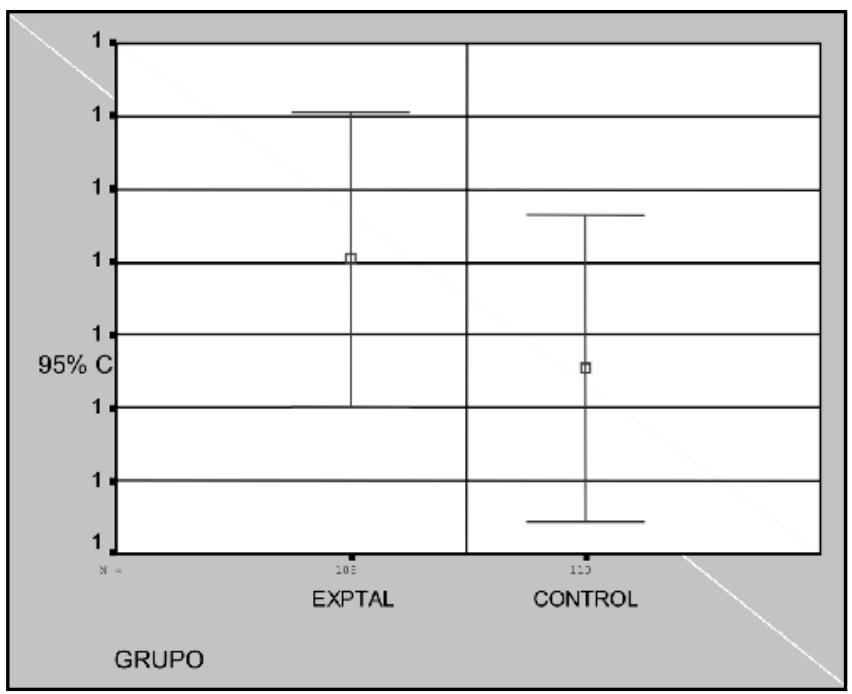

Figura 6. Diagrama comparativo en lechones de valores de IDRC entre grupos experimentales y controles de las dos granjas. 


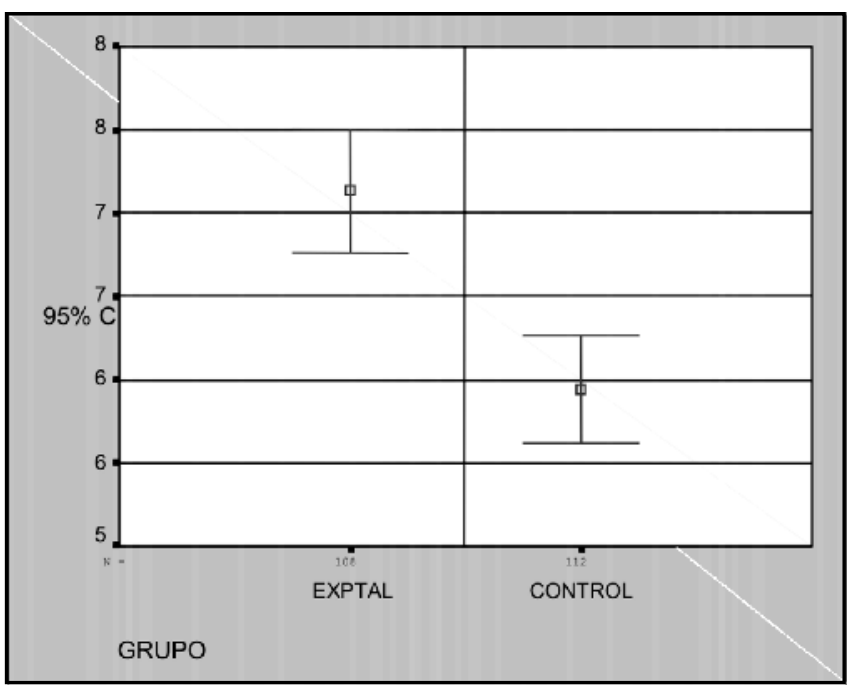

Figura 7. Diagrama comparativo en lechones de valores de la prueba de sulfato de zinc entre grupos experimentales y controles de las dos granjas.

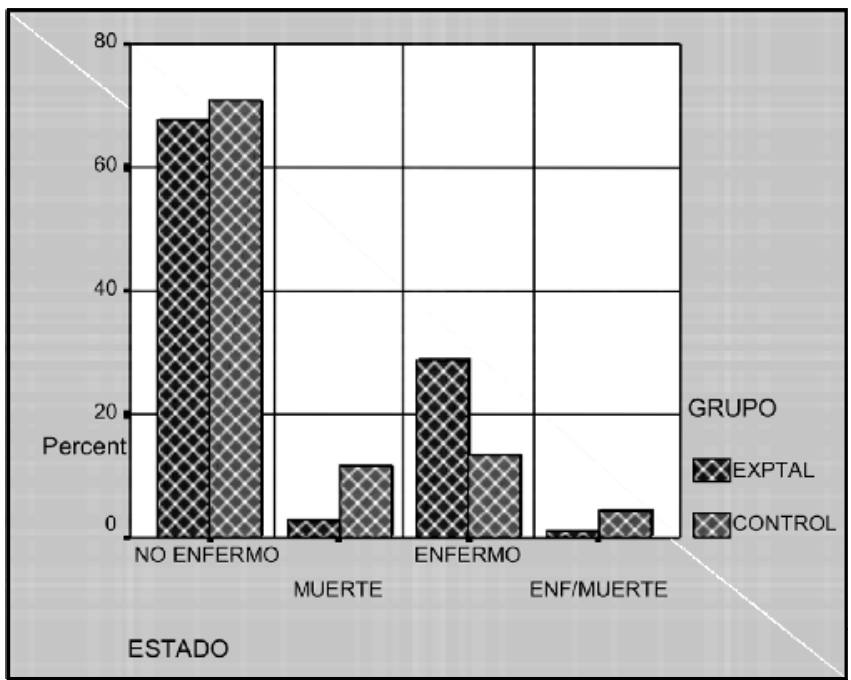

Figura 9. Histograma que muestra el estado de los lechones en los grupos experimentales y controles de las dos granjas.

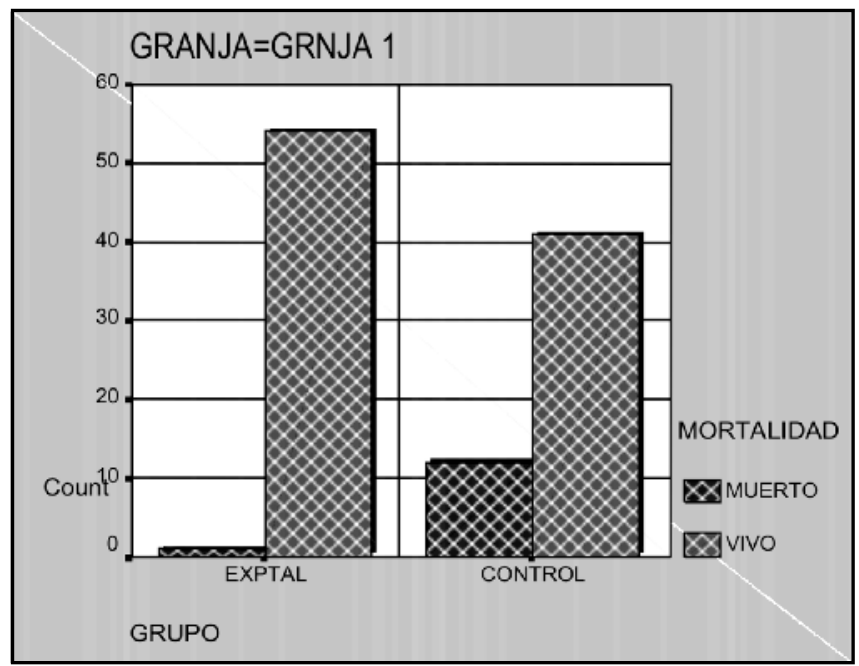

Figura 11. Histograma comparativo de datos en lechones en los grupos experimentales y controles de la granja 1.

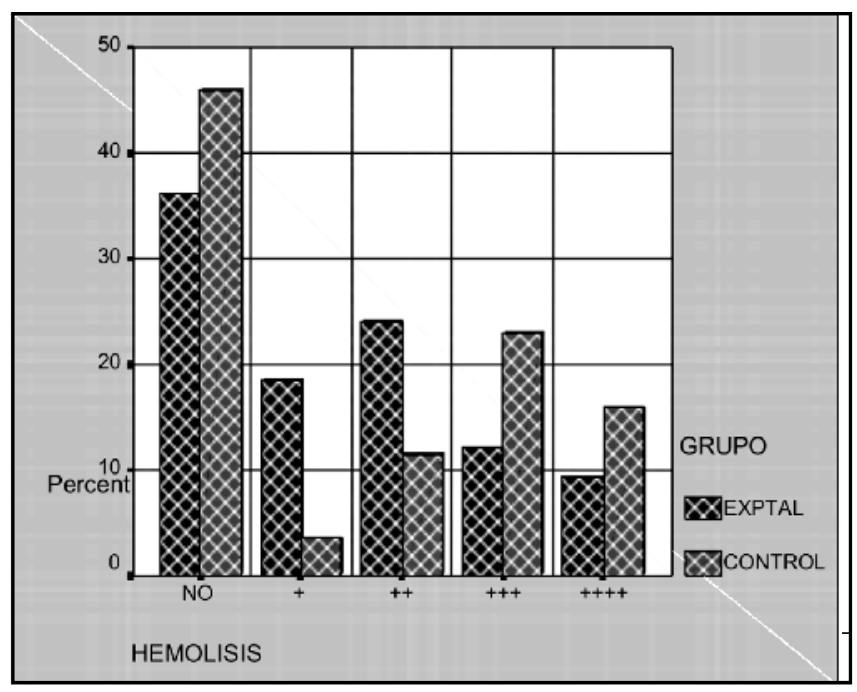

Figura 8. Histograma de la distribución de hemólisis en muestras de lechones de grupos experimentales y controles de las dos granjas.

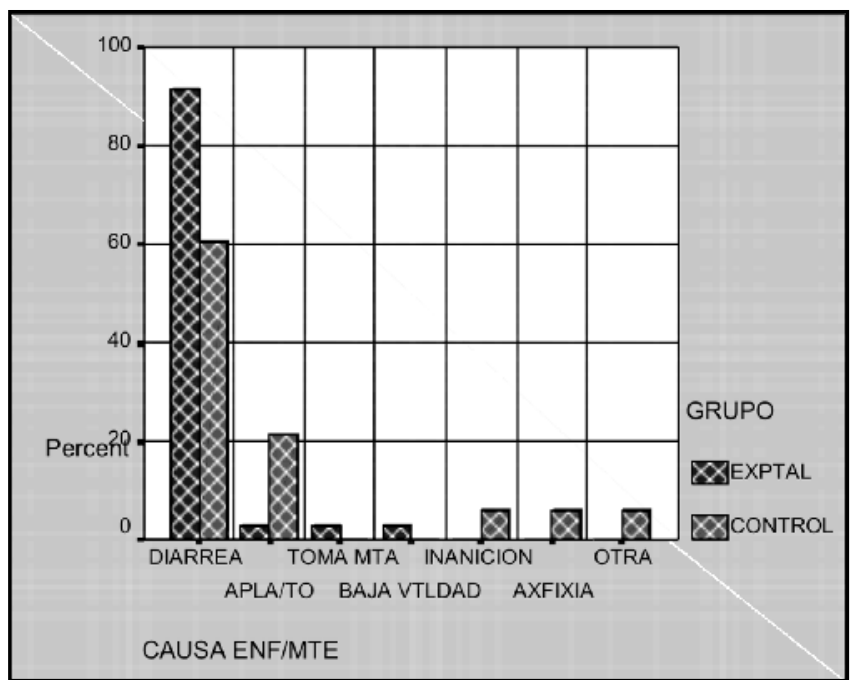

Figura 10. Histograma que muestra las causas de enfermedad y muerte en lechones de los grupos experimentales y controles de las dos granjas.

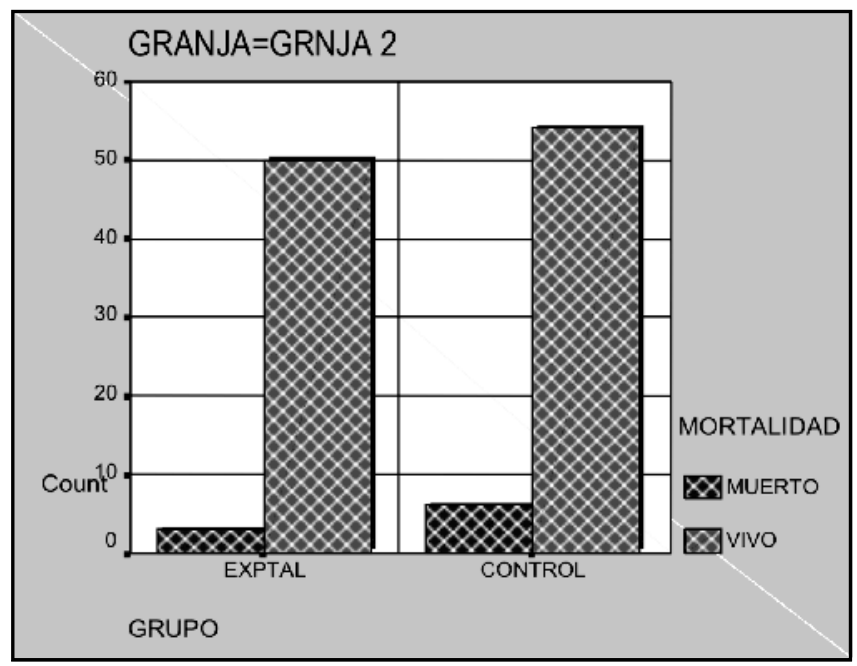

Figura 12. Histograma comparativo de datos en lechones en los grupos experimentales y controles de la granja 2 . 
relativamente homogéneas las lecturas no poseen la sensibilidad necesaria para separarlas.

La prueba de IEF evidenció diferencias apreciadas previamente a través de otras técnicas, en cuanto a concentración de $\mathrm{IgG}$ en los sueros de lechones estudiados.

La hemólisis propiciada por la dificultad que presenta tomar muestras de 2.5 a $3.0 \mathrm{ml}$ de sangre a animales tan pequeños en quienes no se evidencian los vasos sanguíneos, constituyó un inconveniente, que a pesar de no incidir de manera importante en los resultados obtenidos como se mostró gracias a los análisis reportados en el aparte "resultados", si impidió utilizar más profundamente la capacidad clínica de la electroforesis sobre membranas de acetato de celulosa (EAC). Es posible sin embargo, que se puedan establecer curvas de referencia de hemólisis, en cuya realización se laven eritrocitos porcinos en distintas proporciones, se lisen y luego se corran pruebas de EAC para los sobrenadantes obtenidos. Este aspecto, sin embargo, sobrepasaba los límites del presente trabajo.

A pesar de que los lechones de las madres tratadas presentaban mayores pesos al nacimiento y al destete, así como una mayor concentración en suero de Igs pasivas, lo cual tiene una correlación plenamente establecida con resistencia a enfermedades infecciosas hasta el momento del destete como lo reporta Tizard, I. (2), en el presente trabajo se muestra una mayor prevalencia de diarreas en estos animales. Es importante hacer notar que los lechones de las hembras tratadas presentaron menos mortalidad (Figuras 11 y 12), lo cual conduce a tres posibles hipótesis. La primera, es que los animales en realidad no presentan diarrea, pero el excesivo celo de los cuidadores hace que se reporten y traten todos los animales que presenten sobre su región posterior (calzón) residuos de materia fecal líquida, con lo cual, muchos animales que en realidad no presentan diarrea pero que se sentaron sobre excrementos de la hembra o de sus hermanos sean reportados como diarréicos. La segunda es que en realidad si se presentó diarrea pero gracias a la inmunidad pasiva natural más sólida, pudieron sortearla sin mostrar mortalidad. La tercera hace referencia a la calidad inmunológica del calostro consumido, pues no solo es necesario que se transfieran inmunoglobulinas desde la madre hacia sus lechones para que estos sean protegidos, es indispensable que estas inmunoglobulinas sean específicas contra los agentes que causan la problemática infecciosa en los neonatos. Vale resaltar aquí, la importancia adicional que tienen las normas de manejo, higiene y bioseguridad en ese contexto.

Se observó una diferencia apreciable en cuanto a mortalidad entre las dos granjas y de mayor magnitud en los grupos control (grupo experimental 3,7\%, grupo control $15,9 \%$ ) lo cual está de acuerdo con la hipótesis planteada, con la literatura consultada y con los demás resultados obtenidos.

Las hembras se comportaron de manera homogénea en cuanto a las variables analizadas. No hubo diferencias significativas entre los grupos, las granjas o las concentraciones de las distintas fracciones de proteína estudiadas por las diferentes técnicas empleadas.

Los resultados obtenidos permiten inferir que el uso de los inmunomoduladores de la naturaleza del estudiado, pueda ser de utilidad para incrementar los niveles de Igs transferidas pasivamente desde las madres y disminuir la morbimortalidad neonatal relacionada con la presencia de agentes infecciosos en las granjas porcícolas.

\section{Agradecimientos}

A la corporación Colombiana de Investigación Agropecuaria- CORPOICA y a Laboratorios Calier de los Andes S.A. Bogotá, Colombia, por su colaboración y aportes para la realización de este trabajo. 


\section{Referencias}

1. Alfonso, M. y Almansa, J. Concentración de inmunoglobulinas y correlación con salud en neonatos bovinos del pie de monte llanero. Pregrado. Universidad de la Salle. Facultad de Medicina Veterinaria. Bogotá D.C. Colombia. 2001.80p.

2. Almansa, J. y Mariño, O. Fundamentos de la aplicación clínica de estudios electroforéticos sobre membranas de acetato de celulosa. Revista Acovez. 1995 Vol. 20 (1).35-39p.

3. Almansa, J. y López, C. Inmunomoduladores endógenos y exógenos. Revista U.D.C.A. Universidad de ciencias aplicadas y ambientales. Actualidad y divulgación científica. 2000; (2). Bogotá. Colombia.43-51p.

4. Álvarez, B. Análisis de los efectos in vitro del compuesto M1-104 (Inmodulen R) sobre diversos parámetros de la respuesta inmune del cerdo .XVIII Simposium ANAPORC. 1997.

5. Burrin, D., Davis T., Ebner, S., Schoknecht, P., Fiorotto M. and Reeds, P.1997. Colostrum Enhances the nutritional Stimulation of Vital Organ Protein Synthesis in Neonatal Pigs. The journal of Nutrition. 1997 ; 127. (7): 1284-1289.

6. Fiorentino, S., Gutiérrez, M. F., Rueda, N. S. y Rodríguez, J. A. La inmunología en el diagnóstico clínico. Primera edición. Centro Editorial Javeriano, CEJA. Bogotá, Colombia. 1994. 200p.

7. Flórez, H. 2000. Importancia y uso del calostro en bovinos. Información técnica, Corpoica- Regional 8. Año 4 Número 30. Villavicencio, Meta, Colombia. 2000. 8 p.

8. Jensen, A., Elnif, J., Burrin, D. and Sangild, P. 2001. Development of Intestinal Inmunoglobulin Absorption and Enzyme Activities in Neonatal Pigs Is Diet Dependent. Journal of Nutrition. 2001. 131: 3259-3265.

9. Jiang, R., Chang, X., Stoll, B., Ellis, K., Shypailo, R., Weaver, E., Campbell, J. and Burrin, D. Dietary Plasma Protein is Used More Efficiently than Extruded Soy Protein For Lean Tissue Growth in Early Weaned Pigs. Journal of Nutrition. 2000. 130: 2016-1019.

10. Joling, P., Mok, K. S., de Vries Reilingh, G., Wever, P. J. M., Cornelis, R. S., Oskam, J.P. M. y Henken, A. M. An evaluation of immune competence in different swine breeds. Veterinary Quartely. 1993. 15: 9-15.

11. Kawai, T. Proteínas plasmáticas. Aplicación clínica. Editorial médica panamericana S.A. Traducción por Mario A. marino. Buenos Aires, Argentina. 1997. p. 19-121.

12. Lamm,M. Interaction of antigens and antibodies at mucosal surfaces. Annu. Rev. Microbiol. 1997. 51:311-340.

13. Losso, J., Kummer, A., Li-Chan, E. and Nakai, S. Development of a Particle Concentration Fluorescence Immunoassay for the Quantitative Determination of IgG in Bovine Milk. Journal of Agricultural and Food Chemistry. 1993. 41, (4). 682-686.

14. Morilla, A. y Bautista, C. Manual de inmunología. Primera edición. Editorial Diana S. A. México D. F.1986. 433p.

15. Ortiz, J. M. Niveles de Ig A en el calostro de cerdas tratadas con Inmodulen. Laboratorios Calier S.A. 1997.

16. Rietschel, E. y Brade H. Endotoxinas bacterianas. Revista Investigación y ciencia. Edición española de Scientific American. 1992. 193: 16-24.

17. Rodríguez, G., González, G. y Mariño, O. Manual de técnicas en microbiología. Documento de trabajo $\mathrm{N}^{\circ} 18$. Instituto Colombiano Agropecuario ICA, Bogotá,DC., Colombia. 1978. $506 \mathrm{p}$

18. Schneider,W. y Berndt, H. Inmunoelectroforesis. Método y Atlas. Editorial Médica Panamericana. Traducción por Hormaechea, T \& Brainin, K. Buenos Aires, Argentina.1973. $93 \mathrm{p}$.

19. Tizard, I. Inmunología Veterinaria. Sexta edición. Editorial McGraw-Hill Interamericana. Traducción por Palacios, R. Universidad Autónoma de Baja California, México. 2002. $227 \mathrm{p}$.

20. Weir, D. M. 1973. Inmunochemistry. Handbook of experimental Immunology. Segunda edición. Blackwell Scientific Publications. Osney Mead. Oxford. 1973. 19.10-19.12p.

21. Winkelman, N. Peak Immunity Keeps Pigs Well. National Hog Farmer. 1989. Fall. 7-15. 